health than they have, whereas the fraction of women in very good health is higher on the original than on the adjusted scale. In other words, these findings suggest that men underreport and women over-report good health.

Conclusion Our preliminary findings challenge the prevailing assumption of women over-reporting and men under-reporting health problems and highlight the importance to attend to health problems reported by women and men equally carefully.

\section{P36 CANCER PREVENTION IN VULNERABLE WOMEN: AN EXPLORATORY QUALITATIVE STUDY WITH WOMEN WHO HAVE EXPERIENCED DOMESTIC VIOLENCE AND OFFENDING BEHAVIOURS}

${ }^{1} \mathrm{~S}$ Hanson, ${ }^{2} \mathrm{D}$ Gilbert, ${ }^{3} \mathrm{R}$ Landy, ${ }^{4} \mathrm{G}$ Okoli, ${ }^{5} \mathrm{C}$ Guell ${ }^{*}$. School of Health Sciences, University of East Anglia, Norwich, UK; ${ }^{2}$ Oncology, University College London, London, UK; ${ }^{3}$ Centre for Cancer Prevention, Mary University London, London, UK; ${ }^{4}$ Department of Primary Care and Public Health Sciences, King's College London, London, UK; ${ }^{5}$ European Centre for Environment and Human Health, University of Exeter, Truro, UK

\subsection{6/jech-2018-SSMabstracts. 162}

Background Cancer is more common among disadvantaged communities. Women who have experienced domestic violence or offending behaviours are at higher risk of cancer through multiple risk factors: addictive behaviours, low levels of activity and poor screening uptake. Such women are also likely to come from socially marginalised backgrounds where persistent health inequity remains. Addressing their needs is an important factor in cancer prevention and early diagnosis. This exploratory study aimed to explore women's health behaviours in terms of excessive alcohol consumption, tobacco use, physical activity, diet, weight and cancer screening within their context and experiences of vulnerability. We also aimed to assess perceptions of risk to cancer and barriers to health promoting activities.

Methods We conducted 14 semi-structured interviews and two focus groups with vulnerable women attending support groups in a Housing Association women's centre, and seven interviews with the staff who support them. Verbatim transcripts were analysed thematically.

Results We identified three themes: risk factors as markers of distress, inhibiting views of self, and navigating the healthcare system. Risk factors of alcohol use, smoking, physical inactivity and unhealthy eating were common but reported in context of distressing experiences of mental ill-heath, poverty, addition and abuse. Walking, for example, was reported as the result of lost driving licences or a symptom of anxiety; smoking was reported as part of other additive behaviour such as alcohol abuse and drug taking. Women's views of themselves such as self-worth were often negative, shaped by experiences of neglect and abuse, or of perceived negative treatment by social and mental health services. This shaped their trust in health services, as well as low perception of risk for cancer susceptibility and potential for delay in presenting with cancer symptoms. Women frequently reported fatalistic attitudes to cancer, chronic diseases and early death but paradoxically also reported high levels of screening uptake. Despite narratives of distress, women showed self-reliance and resilience, and this might have helped navigating the health systems to some degree, for example using screening services. Women and staff were receptive to health promotion in cancer prevention.
Conclusion Women in this study were at high risk of chronic diseases, including cancer. Their experiences of social disadvantage and personal and structural violence profoundly shaped their practices, aspirations and attitudes towards risk, health and healthcare. Our findings will inform the design of a feasibility study to test a cancer prevention strategy co-designed by and tailored to vulnerable women.

\section{P37 RELATIONSHIP BETWEEN PHYSICAL ACTIVITY AND BLOOD GLUCOSE MARKERS DURING PREGNANCY AMONGST A MULTI-ETHNIC MATERNAL COHORT: RESULTS FROM THE BORN IN BRADFORD COHORT STUDY}

${ }^{1}$ ES Petherick*, ${ }^{1} \mathrm{~L}$ Sherar, ${ }^{2} \mathrm{~S}$ Barber, ${ }^{1} \mathrm{M}$ Hamer, ${ }^{2} \mathrm{~J}$ Wright. ${ }^{1}$ School of Sport, Exercise and Health Sciences, Loughborough University, Loughborough, UK; ${ }^{2}$ Born in Bradford, Bradford Institute for Health Research, Bradford, UK

\subsection{6/jech-2018-SSMabstracts. 163}

Background Physical activity is associated with positive health biomarker profiles during pregnancy and resultant birth outcomes. Furthermore, there is some evidence to suggest that physical activity both prior to and during pregnancy may reduce the risk of gestational diabetes. In this study we aim to investigate the relationship between physical activity and biomarkers associated with gestational diabetes (GDM) risk and clinical diagnoses of GDM.

Methods Participants were pregnant women recruited to the Born in Bradford cohort study who completed phase 1 or 2 of the baseline questionnaire and provided a fasting blood sample between approx. 26-28 weeks of gestation, and had a singleton birth. Where mothers had two births during the study period, only the first was included in the present analyses. Physical activity level was measured using the General Practice Physical Activity Questionnaire (GPPAQ) and questions about usual walking speed. The relationship of selfreported physical activity, using both of the two definitions above, with levels of fasting blood glucose was evaluated using multivariate linear regression. GDM risk was assessed using logistic regression analyses. Models were adjusted for other key covariables including age, ethnicity, body mass index and parity.

Results Data were available for 6119 maternal participants. White British women reported higher levels of physical activity and physical function (i.e. higher self-reported average walking speed) relative to their Pakistani and Other ethnic group peers. Despite the higher GPPAQ scores in the White British group, over 67 per cent still fell into the inactive or moderately inactive category.

Higher levels of activity as measured by GPPAQ scores was not associated with fasting blood glucose or odds of gestational diabetes. Walking speeds were associated with lower levels of fasting glucose, and remained so after adjustment for other relevant covariables $(-0.04(-0.08,-0.05)$. Adjusted odds of gestational diabetes diagnosis were also lower in those reporting the highest self-reported walking speeds OR 0.49 (0.27-0.87).

Conclusion Faster self-reported walking speeds, which may result from greater residual fitness prior to pregnancy, were shown to be associated with lower fasting blood glucose levels and lower odds of a gestational diabetes diagnosis. These 